\title{
INVERSE PROBLEMS FOR SELF-ADJOINT DIRAC SYSTEMS: EXPLICIT SOLUTIONS AND STABILITY OF THE PROCEDURE
}

\author{
ALEXANDER SAKHNOVICH
}

Abstract. A procedure to recover explicitly self-adjoint matrix Dirac systems on the semi-axis (with both discrete and continuous components of spectrum) from rational Weyl functions is considered. Its stability is proved. GBDT version of Bäcklund-Darboux transformation and various important results on Riccati equations are used for this purpose.

Mathematics subject classification (2010): 15A24, 34A55, 34B20, 34D20, 93B20.

Keywords and phrases: Inverse problem, stability, Dirac system, Weyl function, minimal realization, explicit solution, Riccati equation.

\section{REFERENCES}

[1] D. Alpay AND I. GohBerg, Inverse spectral problem for differential operators with rational scattering matrix functions, J. Differential Equations 118 (1995), 1-19.

[2] D. Alpay, I. Gohberg, M. A. KaAshoek, L. Lerer, And A. Sakhnovich, Krein systems and canonical systems on a finite interval: accelerants with a jump discontinuity at the origin and continuous potentials, Integral Equations Operator Theory 68, 1 (2010), 115-150.

[3] R. Brunnhuber, J. Eckhardt, A. Kostenko, And G. Teschl, Singular Weyl-TitchmarshKodaira theory for one-dimensional Dirac operators, Monatsh. Math. 174 (2014), 515-547.

[4] J. L. Cieslins Ki, An algebraic method to construct the Darboux matrix, J. Math. Phys. 36 (1995), 5670-5706.

[5] J. L. Cieslinski, Algebraic construction of the Darboux matrix revisited, J. Phys. A 42 (2009), 404003.

[6] S. Clark And F. Gesztesy, Weyl-Titchmarsh M-function asymptotics, local uniqueness results, trace formulas, and Borg-type theorems for Dirac operators, Trans. Amer. Math. Soc. 354 (2002), 3475-3534.

[7] S. Clark And F. GeszTESy, On Self-adjoint and J-self-adjoint Dirac-type operators: a case study, Contemp. Math. 412 (2006), 103-140.

[8] M. M. CRUM, Associated Sturm-Liouville systems, Quart. J. Math., Oxford II Ser. 6 (1955), 121-127.

[9] P. A. DEIFT, Applications of a commutation formula, Duke Math. J. 45 (1978), 267-310.

[10] J. Eckhardt, F. Gesztesy, R. Nichols, A. Sakhnovich, and G. Teschl, Inverse spectral problems for Schrödinger-type operators with distributional matrix-valued potentials, Differential Integral Equations 28 (2015), 505-522.

[11] B. Fritzsche, M. A. KaAshoek, B. Kirstein, and A. L. Sakhnovich, Skew-self-adjoint Dirac systems with rational rectangular Weyl functions: explicit solutions of direct and inverse problems and integrable wave equations, Math. Nachr. (2016), DOI 10.1002/mana.201500069.

[12] B. Fritzsche, B. Kirstein, I. YA. Roitberg, AND A. L. SAKHnOVICH, Skew-self-adjoint Dirac system with a rectangular matrix potential: Weyl theory, direct and inverse problems, Integral Equations Operator Theory 74, 2 (2012), 163-187.

[13] B. Fritzsche, B. Kirstein, I. YA. Roitberg, And A. L. SAKHnovich, Weyl theory and explicit solutions of direct and inverse problems for a Dirac system with rectangular matrix potential, Oper. Matrices 7, 1 (2013), 183-196.

[14] B. Fritzsche, B. Kirstein, I. Ya. Roitberg, and A. L. Sakhnovich, Discrete Dirac system: rectangular Weyl functions, direct and inverse problems, Oper. Matrices 8, 3 (2014), 799-819. 
[15] B. Fritzsche, B. Kirstein, I. YA. Roitberg, And A. L. SAKhnovich, Skew-selfadjoint Dirac systems: stability of the procedure of explicit solving the inverse problem, arXiv:1510.00793.

[16] B. Fritzsche, B. KIRSTEIn AND A. L. SAKHNOVICH, Completion problems and scattering problems for Dirac type differential equations with singularities, J. Math. Anal. Appl. 317 (2006), 510-525.

[17] B. Fritzsche, B. Kirstein, And A. L. SAKhnovich, Semiseparable integral operators and explicit solution of an inverse problem for the skew-self-adjoint Dirac-type system, Integral Equations Operator Theory 66 (2010), 231-251.

[18] F. GeszTESY, A complete spectral characterization of the double commutation method, J. Funct. Anal. 117 (1993), 401-446.

[19] F. Gesztesy And G. Teschl, On the double commutation method, Proc. Amer. Math. Soc. 124 (1996), 1831-1840.

[20] I. Gohberg, M. A. KAAShoek, AND A. L. SAKhnovich, Canonical systems with rational spectral densities: explicit formulas and applications, Mathematische Nachr. 194 (1998), 93-125.

[21] I. Gohberg, M. A. KAAshoek, AND A. L. SAKhnovich, Pseudocanonical systems with rational Weyl functions: explicit formulas and applications, J. Differential Equations 146, 2 (1998), 375-398.

[22] I. Gohberg, M. A. KAAShoek, And A. L. SAKhnovich, Bound states for canonical systems on the half and full line: explicit formulas, Integral Equations Operator Theory 40, 3 (2001), 268-277.

[23] I. Gohberg, M. A. KAAShoek, And A. L. SAKhnovich, Scattering problems for a canonical system with a pseudo-exponential potential, Asymptotic Analysis 29 (2002), 1-38.

[24] R. O. HRYNIV, Analyticity and uniform stability in the inverse spectral problem for Dirac operators, J. Math. Phys. 52 (2011), 063513.

[25] M. A. KaAShoek And A. L. SaKhnovich, Discrete pseudo-canonical system and isotropic Heisenberg magnet, J. Funct. Anal. 228 (2005), 207-233.

[26] R. E. Kalman, P. Falb, And M. ARbib, Topics in mathematical system theory, International Series in Pure and Applied Mathematics, McGraw-Hill Book Company, New York etc., 1969.

[27] A. Kostenko, A. Sakhnovich, And G. Teschl, Commutation methods for Schrödinger operators with strongly singular potentials, Math. Nachr. 285 (2012), 392-410.

[28] M. G. KREIN, Continuous analogues of propositions on polynomials orthogonal on the unit circle (Russian), Dokl. Akad. Nauk SSSR 105 (1955), 637-640.

[29] M. G. KREIN, On a continuous analogue of a Christoffel formula from the theory of orthogonal polynomials (Russian), Dokl. Akad. Nauk SSSR 113 (1957), 970-973.

[30] P. Lancaster and L. Rodman, Algebraic Riccati equations, Clarendon Press, Oxford, 1995.

[31] H. LAnger, A. C. M. RAN, And D. Temme, Nonnegative solutions of algebraic Riccati equations, Lin. Alg. Appl. 261 (1997), 317-352.

[32] B. M. LEVITAN AND I. S. SARgSJAn, Introduction to the spectral theory. Selfadjoint differential operators, Transl. Math. Monographs 34, Amer. Math. Soc., Providence, RI, 1975.

[33] V. B. Matveev And M. A. SAlle, Darboux transformations and solitons, Springer, Berlin, 1991.

[34] A. Mercado, A. Osses, And L. Rosier, Carleman inequalities and inverse problems for the Schrödinger equation, C. R. Math. Acad. Sci. Paris 346, 1-2 (2008), 53-58.

[35] YA. V. MYKYTYUK AND D. V. PUYDA, Inverse spectral problems for Dirac operators on a finite interval, J. Math. Anal. Appl. 386 (2012), 177-194.

[36] A. C. M. RAN AND L. Rodman, Stability of invariant maximal semidefinite subspaces, II: Applications: selfadjoint rational matrix functions, algebraic Riccati equations, Linear Algebra Appl. 63 (1984), 133-173.

[37] A. L. SAKHNOVICH, Nonlinear Schrödinger equation on a semi-axis and an inverse problem associated with it, Ukr. Math. J. 42, 3 (1990), 316-323.

[38] A. L. SAKhnovich, Exact solutions of nonlinear equations and the method of operator identities, Lin. Alg. Appl. 182 (1993), 109-126.

[39] A. L. SAKHnOVICH, Skew-self-adjoint discrete and continuous Dirac-type systems: inverse problems and Borg-Marchenko theorems, Inverse Problems 22 (2006), 2083-2101.

[40] A. L. SAKHNOVICH, On the GBDT version of the Bäcklund-Darboux transformation and its applications to linear and nonlinear equations and spectral theory, Math. Model. Nat. Phenom. 5 (2010), 340-389.

[41] A. L. SAKHNOVICH, Inverse problem for Dirac systems with locally square-summable potentials and rectangular Weyl functions, J. Spectr. Theory 5, 3 (2015), 547-569. 
[42] A. L. Sakhnovich, A. A. Karelin, J. Seck-Tuoh-Mora, G. Perez-Lechuga, and M. GONZALEZ-HERNANDEZ, On explicit inversion of a subclass of operators with D-difference kernels and Weyl theory of the corresponding canonical systems, Positivity 14 (2010), 547-564.

[43] A. L. SAKHNOVICH, L. A. SAKhNOVICH, AND I. YA. RoITBERG, Inverse problems and nonlinear evolution equations. Solutions, Darboux matrices and Weyl-Titchmarsh functions, De Gruyter Studies in Mathematics 47, De Gruyter, Berlin, 2013.

[44] L. A. SakHNovich, On the factorization of the transfer matrix function, Sov. Math. Dokl. 17 (1976), 203-207.

[45] L. A. SAKhnovich, Factorisation problems and operator identities, Russian Math. Surv. 41 (1986), $1-64$.

[46] G. TESCHL, Deforming the point spectra of one-dimensional Dirac operators, Proc. Amer. Math. Soc. 126 (1998), 2873-2881.

[47] V. E. ZaKharov AND A. V. Mikhailov, On the integrability of classical spinor models in twodimensional space-time, Commun. Math. Phys. 74 (1980), 21-40. 